\title{
Microbiome in atopic dermatitis
}

This article was published in the following Dove Press journal:

Clinical, Cosmetic and Investigational Dermatology

22 February 2017

Number of times this article has been viewed

\section{Uwe Wollina \\ Department of Dermatology and Allergology, Academic Teaching Hospital Dresden-Friedrichstadt, Dresden, Germany}

Correspondence: Uwe Wollina Department of Dermatology and Allergology, Academic Teaching Hospital Dresden-Friedrichstadt, Friedrichstrasse 4I, 01067 Dresden, Germany

Tel +4945 I 480 I685

Fax +49 35। 480 I2I9

Email wollina-uw@khdf.de
Abstract: Atopic dermatitis (AD) is a common chronic inflammatory skin disease affecting $\sim 10-20 \%$ of the general population. $\mathrm{AD}$ is characterized by disturbances in epidermal barrier function and hyperactive immune response. Recently, changes in the skin and intestinal microbiome have been analyzed in more detail. The available data suggest a link between disturbed skin microbiome and course of the disease. Flares of the disease are associated with an expansion of Staphylococcus aureus on lesional skin and a substantial loss of biodiversity in skin microbiome. Staphylococci exoproteins and superantigens evoke inflammatory reactions in the host. Skin microbiome includes superficial stratum corneum that is affected by environmental factors such as exposure to germs and cleansing. Available evidence argues for a link between epidermal barrier impairment and disturbances in skin microbiome in AD. In contrast to skin microbiome, intestinal microbiome seems to become stabilized after infancy. There is also a significant heritable component for intestinal microbiome. The microbial taxa, relative percentages and quantities vary remarkably between the different parts of the intestinal tract. Early intestinal microbial colonization may be a critical step for prevention of further development of AD. Skin barrier-aimed topical treatments help to develop a neo-microbiome from deeper compartments. Probiotics, prebiotics and synbiotics have been investigated for the treatment of $\mathrm{AD}$, but further investigations are needed. Targeted treatment options to normalize skin and intestinal microbiome in $\mathrm{AD}$ are under investigation.

Keywords: atopic dermatitis, microbiome, staphylococci, skin, intestine, antimicrobial peptides

\section{Introduction}

On skin and mucous membranes (intestine, airways), microbial genomes outnumber those of the human host by a factor of 100 . The microbiome (microbiota), i.e., the bacteria, viruses, archaea and fungi, living on and within the human body contributes to health and disease. The microbiome is individual and changes with age. The crosstalk between the microbiome and the human host is realized by secretion of metabolites from microbes and the human immune system scanning the microbiome for information about metabolic state and colonization. Thereby, bacteria-derived molecules such as short-chain fatty acids may influence human epigenomic pathways. ${ }^{1,2}$ This article reviews the role of the human microbiome in atopic dermatitis (AD).

\section{AD}

AD is a common chronic inflammatory skin disease affecting $\sim 20 \%$ of children. In $95 \%$ of cases, initial manifestation of AD occurs within the first 5 years of life. 
Approximately a quarter of these children continue to have AD during adulthood. ${ }^{3}$

In the pre-school age, $30 \%$ of children with $\mathrm{AD}$ suffer from food allergies (eggs, cow's milk, peanuts). Those with moderate to severe $\mathrm{AD}$ have a $50 \%$ risk of developing asthma and $75 \%$ risk of developing hay fever. ${ }^{4,5}$

AD diagnosis is made by almost exclusively clinical criteria. ${ }^{6-8}$ Genetic and epigenetic factors modulate AD. Environmental factors such as (perinatal) exposure to indoor and outdoor allergens and pollutants, nutrition and microbiome are considered as influential for the manifestation and severity of AD. Environmental factors regulate gene expression through microRNA (miR) and genomic DNA modification. Among genetic factors, filaggrin (FLG) null gene mutations are the most significant risk factor for AD. Furthermore, genes in T helper lymphocyte type 2 (Th2) signaling pathways represent a second important genetic risk factor for $\mathrm{AD}$, although predominant Th2 response is limited to the flare-ups. Genome-wide association studies have identified $>30$ risk loci for AD for genes involved in epidermal barrier function and immune response. Gene profiling assays revealed overexpression of Th2, Th17 and miR-155.,10

Epigenetic studies in AD have demonstrated significant changes in the methylation status of skin lesions, e.g., hypomethylation of gene promoters and alterations in $\mathrm{miR}$ profile. ${ }^{11-13}$ Genetic and epigenetic studies suggest that two major pathways are involved in $\mathrm{AD}$, i.e., innate and adaptive immune systems and epidermal barrier function. ${ }^{9-13}$

Levels of the antimicrobial metabolite sphingosine and antimicrobial peptides cathelicidin and defensins B2 and B3 are reduced in the skin of AD subjects..$^{14}$ These factors contribute to the increased risk of cutaneous infections in AD.

\section{Cutaneous microbiome and AD}

Resident skin bacteria are influenced by topological and endogenous factors of skin and can be modulated by external factors such as clothing, hygiene, topical treatments and skin care products (Table 1). There are gender differences in skin microbiome as well. Skin microbiomes differ between children and adults (described in the following). Bacteria are not uniformly distributed in skin. There is a superficial and a deeper compartment in the human stratum corneum. After injury, a neo-microbiome is produced from the deeper compartment, which can be regarded as the indigenous microbiome. Furthermore, bacteria are consistently detectable also in deeper skin layers such as the dermis and the subcutaneous adipose tissue. A balanced resident skin flora is a protective measure. ${ }^{15,16}$
Table I Normal human skin microbiome ${ }^{a}$

\begin{tabular}{|c|c|}
\hline Skin type & $\begin{array}{l}\text { Normal microbiome } \\
\text { (most abundant bacterial groups) }\end{array}$ \\
\hline Sebaceous skin & $\begin{array}{l}\text { Propionibacteria spp., Corynebacteria spp., } \\
\text { other Actinobacteriales spp., Staphylococci spp. }\end{array}$ \\
\hline Moist skin & $\begin{array}{l}\text { Corynebacteria spp., Staphylococci spp., } \\
\beta \text {-Proteobacteria, } \gamma \text {-Proteobacteria }\end{array}$ \\
\hline Dry skin & $\begin{array}{l}\beta \text {-Proteobacteria, Corynebacteria spp., } \\
\text { Flavobacteriales }\end{array}$ \\
\hline
\end{tabular}

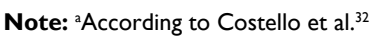

Among the Gram-positive Staphylococcus species, Staphylococcus epidermidis is the dominant type in healthy skin with the ability to inhibit the growth of Staphylococcus aureus. ${ }^{17}$ In children, colonization of skin by $S$. epidermidis and $S$. cohnii during the first year of life has a protective effect on the development of AD. ${ }^{18}$

Disturbances in cutaneous microbiome represent an independent risk factor for the development of AD. In $\sim 90 \%$ of patients suffering from $\mathrm{AD}$, the skin becomes colonized by S. aureus of which $50 \%$ are toxin producing. These toxins can contribute to inflammation and skin barrier dysfunction via activating the host inflammasomes. ${ }^{19}$

Using whole metagenome profiling, distinct signatures enriched for Streptococcus and Gemella but depleted for Dermacoccus were identified in subjects prone to AD. This was accompanied by changes in the innate and Th1 adaptive immune responses to $S$. epidermidis and $S$. aureus. ${ }^{20}$

In lesional AD, however, the proportions of both $S$. aureus and $S$. epidermidis increase. Since these species produce antibacterial compounds such as antimicrobial peptides and bacteriocins, a relative decrease in other species, including Propionibacterium, Corynebacterium and Streptococcus, occurs during AD flares. After successful topical AD treatment, there is an increasing biodiversity of cutaneous microbiome that arises from taxa already present in cutaneous microbiome. ${ }^{21,22}$

In AD, S. aureus is capable of inducing flares of the disease. There is an increased colonization of lesional skin in $\mathrm{AD}$ patients. Membrane vesicles released from these bacteria can penetrate the epidermis and induce a massive infiltration of inflammatory cells with a mixed Th1/Th2 immune response. ${ }^{23}$ S. aureus itself is capable of penetrating the epidermis in case of increased cathelicidin expression and increased expression of interleukin (IL)-4, IL-13, IL-22, and other cytokines. ${ }^{24} S$. aureus can directly impair skin barrier function by stimulating the production of keratinocyte endogenous serine protease. This diminishes FLG and other epidermal proteins and contributes to disturbed lipid lamellar function. ${ }^{25}$ S. aureusassociated, microbial-associated molecular patterns bind to 
Toll-like receptor 2 (TLR2) heterodimers and induce longlasting and self-perpetuating T-cell inflammation. ${ }^{26}$

On the other hand, $\mathrm{AD}$ is a risk factor for colonization of nasal mucous membranes and the skin by methicillinresistant $S$. aureus (MRSA). ${ }^{27}$ MRSA prevalence on lesional skin has been reported from 13 to $24 \%{ }^{28,29}$ This can cause recurrent MRSA infections in patients with $\mathrm{AD} .{ }^{30}$

Staphylococcus epidermidis secretome, on the other hand, promotes the activity of regulatory T-cells (Treg), suppressing the proliferation of CD4-positive T cells. Furthermore, S. epidermidis induces the release of IL-10 by skin dendritic cells. ${ }^{31}$

It becomes obvious that changes in skin microbiome are most critical during early life time, when skin barrier function and immune system are rather immature. Skin microbiome is dynamic since it changes with time. Operational taxonomic unit (OTC) stability of skin microbiome has been found less abundant and therefore more instable than that of intestinal microbiome, probably due to cleaning and other extrinsic factors. ${ }^{32,33}$

\section{Intestinal microbiome and AD}

The fetal intestine becomes colonized before delivery by bacterial transmission from the mother through the placental barrier. The mode of delivery has further impact on intestinal microbiome. It has been demonstrated that delivery by cesarean section decreases the colonization by Bacteroides but increases the number of Clostridiae. ${ }^{34}$

It has been assumed that small intestine permeability may be increased in AD patients. ${ }^{35,36}$ Intestinal permeability is one factor in acquired food allergy. ${ }^{37}$

Disturbances in intestinal microbiome could be a risk factor of further AD development. Intrapartum antibiosis for $>24 \mathrm{~h}$ increased the relative risk for AD infants at the age of 2 years by $1.99 .{ }^{38}$

Staphylococcus aureus was isolated from rectal swabs from infants aged 0 to 2 months. $S$. aureus strains of infants who developed $\mathrm{AD}$ were different from the strains of infants who were not affected at the age of 18 months. A combination of expression of superantigen SEIM and adhesin elastinbinding protein by $S$. aureus was protective for AD. ${ }^{39}$

High fecal calprotectin at the age of 2 months is a risk factor for $\mathrm{AD}$. There is an inverse correlation to intestinal Escherichia coli colonization. It can be concluded that early intestinal colonization by $E$. coli has long-term health implications and is a protective measure for AD. ${ }^{40}$

A South Korean study investigated the intestinal microbiome in $90 \mathrm{AD}$ and 42 non-AD subjects. They observed an enrichment of Faecalibacterium prausnitzii $\mathrm{F} 6$ in AD, most distinct in the youngest patients, leading to a decreased intestinal concentration of butyrate and propionate. ${ }^{41}$

Another study used microarray analysis of intestinal microbiome in infants with and without AD at 6 and 18 months of age. Although the authors did not find significant differences at 6 months, healthy children at 18 months harbored threefold greater number of Bacteroidetes. Infants with AD showed increased numbers of Clostridiae. ${ }^{42}$

Intestinal microbiome is dynamic during the first 3 years of life before stabilizing. ${ }^{43}$ In contrast to skin microbiome, intestinal microbiome demonstrates heritability as demonstrated by a recent metagenomics shotgun sequencing study in adult twins. ${ }^{44}$

\section{Microbiome in the treatment of AD Therapies targeting skin microbiome}

There is much evidence suggesting a link between impairment of epidermal barrier function and disturbed skin microbiome. ${ }^{15,22}$

Antibiotics and antiseptics may decrease skin colonization by $S$. aureus but fail to improve the microbiome. On the other hand, topical treatments with corticosteroids, calcineurin inhibitors or even moisturizers and emollients are capable to restore barrier function and normalize skin microbiome..$^{22,45,46}$

To restore cutaneous microbiome in $\mathrm{AD}$, transplantation of microbiota from healthy volunteers might become an option. Skin culturable Gram-negative microbiome differs between $\mathrm{AD}$ patients and healthy controls. In a mouse model of AD, culturable Gram-negative bacteria such as Roseomonas mucosa from healthy volunteers reduced the growth of $S$. aureus, enhanced skin barrier function and activated innate immune function. ${ }^{47}$ In the future, such a treatment may become available for human patients.

Textiles serve as our second skin. Functional textiles may improve skin quality. Chitosan-coated long-sleeve pyjama tops and pants worn overnight for 8 weeks improved the severity scoring of atopic dermatitis index from baseline in $43.8 \%$ of patients whereas only $16.5 \%$ improved with the uncoated pyjamas. A significant decrease in coagulase-negative Staphylococci was observed on the skin with the chitosan-coated product. ${ }^{48}$

Another open trial investigated the effect of $\mathrm{ZnO}$ fabrics on $\mathrm{AD}$ severity in adult patients. The fabric was used overnight on three consecutive days. $\mathrm{ZnO}$ has antibacterial activity and serves as an oxygen radical scavenger. Severity of $\mathrm{AD}$, pruritus and sleep quality of patients improved even after short-time application of the functional textile. ${ }^{49}$

A meta-analysis evaluated published studies about functional textiles in AD. Fabrics based on silk, silver-coated cotton, borage oil and ethylene vinyl alcohol (EVOH) fibers 
were analyzed. Silk and silver-coated cotton demonstrated the strongest effects on AD severity, but only silver-coated cotton reduced $S$. aureus colonization of skin. ${ }^{50}$

On the other hand, it is questionable whether this attempt would have any impact on dermal and subcutaneous adipose tissues, which are a substantial part of cutaneous microbiome. ${ }^{16}$

\section{Therapies targeting intestinal microbiome}

Probiotics are defined as live microbial food ingredients that confer health benefits for the consumer. ${ }^{51}$ In most AD studies, probiotics have not shown a beneficial effect, independent of the age of $\mathrm{AD}$ patients. ${ }^{52}$ Two recent meta-analyses, however, found best evidence for probiotic supplementation in mothers and infants for Lactobacillus rhamnosus GG in long-term prevention of AD. ${ }^{53,54}$

In a prospective randomized trial, the oral application of Lactobacillus salivarius LS01 and Bifidobacterium breve BR03 for 12 weeks to adult patients with AD improved severity (as measured by the SCORAD index), quality of life (dermatology quality of life index) and ratio of Th17 to Treg cells and diminished immune activation and microbial translocation. ${ }^{55}$

In a mouse model, $\mathrm{AD}$ was improved by oral administration of Lactobacillus casei var. rhamnosus (LCR35), which increased the gut population of Bacteroides fragilis, Lactobacilli, Bifidobacterium and Enterococcus, while Clostridium coccoides became less frequent. The treatment also restored the Th1/Th2 balance. ${ }^{56}$

Oral administration of the probiotic Bifidobacterium animalis subsp lactis (LKM512) alleviated pruritus in adult $\mathrm{AD}$ patients in a placebo-controlled prospective trial. Metabolomic analysis suggested that the antipruritic effect was due to increased production of fecal kynurenic acid. ${ }^{57}$

In an open trial, 130 pregnant women were treated with B. breve M16V and Bifidobacterium longum BB536 1 month before delivery; 36 mother-infant pairs served as controls. After delivery, the infants were treated for 6 months with the same combination of probiotics. The risk of AD was significantly reduced within the first 18 months of life (odds ratio at 18 months: 0.304 ). Temporary changes in intestinal microbiome were noted in infants who developed AD. ${ }^{58}$

A meta-analysis of randomized controlled trials suggested the highest efficacy in reduction of severity of AD and prevention of AD by synbiotics, i.e., a combination of prebiotics and probiotics with a mixture of different microbial strains, in children $>1$ year of age. ${ }^{59}$

Primary prevention of AD in infants by probiotic supplementation of their breast-feeding mothers 2-4 weeks before delivery failed in a randomized, double-blind trial. ${ }^{60}$
Prebiotics are defined as selectively fermented nutrients that cause specific changes in composition and/or activity of intestinal microbiome. ${ }^{61}$ The first randomized controlled trial in infants at high risk for AD included 259 patients during their first 6 months of life. The verum group got a mixture of prebiotic galacto-oligosaccharides and long-chain fructo-oligosaccharides. At the end of this trial, 9.8\% in the verum group and $23.1 \%$ in the placebo group developed $\mathrm{AD}$, demonstrating a preventive effect of prebiotics. On the other hand, severity of AD was not affected by prebiotics. ${ }^{62}$

Two prospective randomized and placebo-controlled trials in infants using either prebiotic galacto-oligosaccharides or a mixture of prebiotics failed to reduce severity of AD. ${ }^{63,64}$ Another trial in low-risk infants suggested a temporary preventive effect on $\mathrm{AD} .{ }^{65}$

Most of these trials observed changes in intestinal microbiome with increased numbers of Bifidobacteria but reduced numbers of Clostridiae. ${ }^{63-65}$

Daily intake of Lactobacillus plantarum YIT 0132-fermented citrus juice (LP0132-fermented juice) alleviated AD symptoms in adults during 8 weeks of treatment and further 8 weeks off treatment in two open trials. ${ }^{66}$

Fecal microbiome transplantation has been successful in some intestinal diseases such as recurrent Clostridium difficile-induced pseudomembranous enterocolitis and Crohn's disease, but data on $\mathrm{AD}$ are still missing. ${ }^{47,67}$

\section{Conclusion}

$\mathrm{AD}$ is a common inflammatory skin disease. Recent investigations suggested a role of skin and intestinal microbiome in AD. Most data have been observed from infants, and those from other age groups are rather limited. Furthermore, data from populations less often affected by AD than Caucasians and Asians are almost nonexistent.

Skin microbiome includes not only superficial stratum corneum that is affected by environmental factors such as exposure to germs and cleansing. Available evidence argues for a link between epidermal barrier impairment and disturbances in skin microbiome in AD.

Until today, studies considering the different compartments/tissue layers populated by skin microbiome in AD have not been investigated in detail.

In contrast to skin microbiome, intestinal microbiome seems to become stabilized after infancy. There is also a significant heritable component for intestinal microbiome. The microbial taxa, relative percentages and quantities vary remarkably between the different parts of the intestinal tract. 
Skin barrier-aimed topical treatments help to develop a neomicrobiome from deeper compartments. Probiotics, prebiotics and synbiotics have been investigated for the treatment of $A D$, but further investigations are needed. Current understanding suggests that there may be a window of time to gain best results before the age of 3 years. Normal delivery and avoidance of antibiotics in the perinatal period seem to have a preventive effect in AD. Targeted treatment options to normalize skin and intestinal microbiome in $\mathrm{AD}$ are under investigation.

\section{Disclosure}

The author reports no conflicts of interest in this work.

\section{References}

1. Egert M, Simmering R. The microbiota of the human skin. Adv Exp Med Biol. 2016;902:61-81.

2. Levy M, Blacher E, Elinav E. Microbiome, metabolites and host immunity. Curr Opin Microbiol. 2016;35:8-15.

3. Williams HC. Clinical practice. Atopic dermatitis. NEngl J Med. 2005; 352(22):2314-2324.

4. Asher MI, Montefort S, Björkstén B, et al; ISAAC Phase Three Study Group. Worldwide time trends in the prevalence of symptoms of asthma, allergic rhinoconjunctivitis, and eczema in childhood: ISAAC phases one and three repeat multicountry cross-sectional surveys. Lancet. 2006;368(9537):733-743.

5. Bingefors K, Svensson A, Isacson D, Lindberg M. Self-reported lifetime prevalence of atopic dermatitis and co-morbidity with asthma and eczema in adulthood: a population-based cross-sectional survey. Acta Derm Venereol. 2013;93(4):438-441.

6. Hanifin JM, Rajka G. Diagnostic features of atopic dermatitis. Acta Derm Venereol Suppl (Stockh). 1980;92:44-47.

7. Williams HC, Burney PG, Hay RJ, et al. The U.K. Working Party's Diagnostic Criteria for atopic dermatitis. I. Derivation of a minimum set of discriminators for atopic dermatitis. Br J Dermatol. 1994;131(3): 383-396.

8. Schram ME, Leeflang MM, DEN Ottolander JP, Spuls PI, Bos JD. Validation and refinement of the Millennium Criteria for atopic dermatitis. J Dermatol. 2011;38(9):850-858.

9. Sandilands A, Terron-Kwiatkowski A, Hull PR, et al. Comprehensive analysis of the gene encoding filaggrin uncovers prevalent and rare mutations in ichthyosis vulgaris and atopic eczema. Nat Genet. 2007; 39(5):650-654.

10. Bin L, Leung DY. Genetic and epigenetic studies of atopic dermatitis. Allergy Asthma Clin Immunol. 2016;12:52.

11. Rodriguez E, Baurecht H, Wahn AF, et al. An integrated epigenetic and transcriptonomic analysis reveals distinct tissue-specific patterns of DNA methylation associated with atopic dermatitis. J Invest Dermatol. 2014;134(7):1873-1883.

12. Lv Y, Qi R, Xu J, et al. Profiling of serum and urinary microRNAs in children with atopic dermatitis. PLoS One. 2014;9(1):e115448.

13. Chen XF, Zhang LJ, Zhang J, et al. MiR-151a is involved in the pathogenesis of atopic dermatitis by regulating interleukin-12 receptor $\beta 2$. Exp Dermatol. Epub 2016 Dec 19:doi: 10.1111/exd.13276.

14. D’Auria E, Banderali G, Barberi S, et al. Atopic dermatitis: recent insights on pathogenesis and novel therapeutic target. Asian Pac J Allery Immunol. 2016;34:98-104.

15. Zeeuwen PL, Boekhorst J, van den Bogaard EH, et al. Microbiome dynamics of human epidermis following skin barrier disruption. Genome Biol. 2012;13(11):R101.

16. Nakatsuji T, Chiang HI, Jiang SB, Nagarajan H, Zengler K, Gallo RL. The microbiome extends to subepidermal compartments of normal skin. Nat Commun. 2013;4:1431.
17. Iwase T, Uehara Y, Shinji H, et al. Staphylococcus epidermidis Esp inhibits Staphylococcus aureus biofilm formation and nasal colonization. Nature. 2010;465(7296):346-349.

18. Kennedy EA, Connolly J, Hourihane JO, et al. Skin microbiome before development of atopic dermatitis: early colonization with commensal staphylococci at 2 months is associated with a lower risk of atopic dermatitis at 1 year. J Allergy Clin Immunol. 2017;139(1):166-172.

19. Park KD, Pak SC, Park KK. The pathogenetic effect of natural and bacterial toxins on atopic dermatitis. Toxins (Basel). 2016;9(1):pii:E3.

20. Chng KR, Tay AS, Li C, et al. Whole metagenome profiling reveals skin microbiome-dependent susceptibility to atopic dermatitis flare. Nat Microbiol. 2016;1(9):16106.

21. Kong HH, Oh J, Deming C, et al; NISC Comparative Sequence Program. Temporal shifts in the skin microbiome associated with disease flares and treatment in children with atopic dermatitis. Genome Res. 2012; 22(5):850-859.

22. Gonzalez ME, Schaffer JV, Orlow SJ, et al. Cutaneous microbiome effects of fluticasone propionate cream and adjunctive bleach baths in childhood atopic dermatitis. JAm Acad Dermatol. 2016;75(3):481-493.

23. Jun SH, Lee JH, Kim SI. Staphylococcus aureus-derived membrane vesicles exacerbate skin inflammation in atopic dermatitis. Clin Exp Allergy. 2017;47(1):85-96.

24. Nakatsuji T, Chen TH, Two AM, et al. Staphylococcus aureus exploits epidermal barrier defects in atopic dermatitis to trigger cytokine expression. J Invest Dermatol. 2016;136(11):2192-2200.

25. Williams MR, Nakatsuji T, Sanford JA, Vrbanac AF, Gallo RL. Staphylococcus aureus induces increased serine protease activity in keratinocytes. J Invest Dermatol. 2017;137(2):377-384.

26. Biedermann T, Skabytska Y, Kaesler S, Volz T. Regulation of $T$ cell immunity in atopic dermatitis by microbes: the Yin and Yang of cutaneous inflammation. Front Immunol. 2015;6:353.

27. Daeschlein G, von Podewils S, Bloom T, et al. Risk factors for MRSA colonization in dermatologic patients in Germany. J Dtsch Dermatol Ges. 2015;13(10):1015-1022.

28. Jung MY, Chung JY, Lee HY, Park J, Lee DY, Yang JM. Antibiotic susceptibility of Staphylococcus aureus in atopic dermatitis: current prevalence of methicillin-resistant Staphylococcus aureus in Korea and treatment strategies. Ann Dermatol. 2015;27(4):398-403.

29. Cavalcante FS, Abad ED, Lyra YC, et al. High prevalence of methicillin resistance and PVL genes among Staphylococcus aureus isolates from the nares and skin lesions of pediatric patients with atopic dermatitis. Braz J Med Biol Res. 2015;48(7):588-594.

30. Ong PY. Recurrent MRSA skin infections in atopic dermatitis. J Allergy Clin Immunol Pract. 2014;2(4):396-369.

31. Laborel-Préneron E, Bianchi P, Boralevi F, et al. Effects of the Staphyloccocus aureus and Staphylococcus epidermidis secretomes isolated from the skin microbiota of atopic children on $\mathrm{CD}^{+} \mathrm{T}$ cell activation. PLoS One. 2015;10(10):e0141067.

32. Costello EK, Lauber CL, Hamady M, Fierer N, Gordon JI, Knight R. Bacterial community variation in human body habitats across space and time. Science. 2009;326(5960):1694-1697.

33. Wilkins D, Leung MH, Lee PK. Microbiotica fingerprints loose individually identifying features over time. Microbiome. 2017;5(1):1.

34. Penders J, Gerhold K, Thijs C, et al. New insights into the hygiene hypothesis in allergic diseases: mediation of sibling and birth mode effects by the gut microbiota. Gut Microbes. 2014;5(2):239-244.

35. Ukabam SO, Mann RJ, Cooper BT. Small intestinal permeability to sugars in patients with atopic eczema. Br J Dermatol. 1984;110(6): 649-652.

36. Pike MG, Heddle RJ, Boulton P, Turner MW, Atherton DJ. Increased intestinal permeability in atopic eczema. J Invest Dermatol. 1986;86(2): 101-104.

37. Isolauri E. Intestinal involvement in atopic disease. J R Soc Med. 1997; 90(suppl 30):S15-S20.

38. Wohl DL, Curry WJ, Mauger D, Miller J, Tyrie K. Intrapartum antibiotics and childhood atopic dermatitis. J Am Board Fam Med. 2015; 28(1):82-89. 
39. Nowrouzian FL, Lina G, Hodille E, et al. Superantigens and adhesins of infant gut commensal Staphylococcus aureus strains and association with subsequent development of atopic eczema. Br J Dermatol. Epub Oct 19 2016:doi: 10.1111/bjd.15138.

40. Orivuori L, Mustonen K, de Goffau MC, et al; PASTURE Study Group. High level of fecal calprotectin at age 2 months as a marker of intestinal inflammation predicts atopic dermatitis and asthma by age 6. Clin Exp Allergy. 2015;45(5):928-939.

41. Song H, Yoo Y, Hwang J, Na Y-C, Kim HS. Faecalibacterium prausnitzii subspecies-level dysbiosis in the human gut microbiome underlying atopic dermatitis. J Allergy Clin Immunol. 2016;137(3):852-860.

42. Nylund L, Satokari R, Nikkilä J, et al. Microarray analysis reveals marked intestinal microbiota aberrancy in infants having eczema compared to healthy children in at-risk for atopic dermatitis. $B M C$ Microbiol. 2013;13:12.

43. Yassour M, Vatanen T, Siljander H, et al. Natural history of the infant gut microbiome and impact of antibiotic treatment on bacterial strain diversity and stability. Sci Transl Med. 2016;8(343):343ra81.

44. Xie H, Guo R, Zhong H, et al. Shotgun metagenomics of 250 adult twins reveals genetic and environmental impacts on the gut microbiome. Cell Syst. 2016;3(6):572-584.

45. Hon KL, Leung AK, Barankin B. Barrier repair therapy in atopic dermatitis: an overview. Am J Clin Dermatol. 2013;14(5):389-399.

46. Fukaya M, Sato K, Yamada T, et al. A prospective study of atopic dermatitis managed without topical corticosteroids for a 6-month period. Clin Cosmet Investig Dermatol. 2016;9:151-158.

47. Myles IA, Williams KW, Reckhow JD, et al. Transplantation of human skin microbiota in models of atopic dermatitis. JCI Insight. 2016;1(10):pii:e86955.

48. Lopes C, Soares J, Tavaria F, et al. Chitosan coated textiles may improve atopic dermatitis severity by modulating skin staphylococcal profile: a randomized controlled trial. PLoS One. 2015;10(11):e0142844.

49. Wiegand C, Hipler UC, Boldt S, Strehle J, Wollina U. Skin-protective effects of a zinc oxide-functionalized textile and its relevance for atopic dermatitis. Clin Cosmet Investig Dermatol. 2013;6:115-121.

50. Lopes C, Silva D, Delgado L, Correia O, Moreira A. Functional textiles for atopic dermatitis: a systematic review and meta-analysis. Pediatr Allergy Immunol. 2013;24(6):603-613.

51. Caramia G, Atzei A, Fanos V. Probiotics and the skin. Clin Dermatol. 2008;26(1):4-11.

52. Vandenplas Y, Huys G, Daube G. Probiotics: an update. J Pediatr (Rio J). 2015;91(1):6-21.

53. Foolad N, Brezinski EA, Chase EP, Armstrong AW. Effect of nutrient supplementation on atopic dermatitis in children: a systematic review of probiotics, prebiotics, formula, and fatty acids. JAMA Dermatol. 2013; 149(3):350-355.
54. Pelucchi C, Chatenoud L, Turati F, et al. Probiotics supplementation during pregnancy or infancy for the prevention of atopic dermatitis: a meta-analysis. Epidemiology. 2012;23(3):402-414.

55. Iemoli E, Trabattoni D, Parisotto S, et al. Probiotics reduce gut microbial translocation and improve adult atopic dermatitis. J Clin Gastroenterol. 2012;46(suppl):S33-S40.

56. Enomoto T, Sowa M, Nishimori K, et al. Effects of bifidobacterial supplementation to pregnant women and infants in the prevention of allergy development and on fecal microbita. Allergol Int. 2014;63(4): 575-585.

57. Yeom M, Sur BJ, Park J, et al. Oral administration of Lactobacillus casei variety rhamnosus partially alleviates TMA-induced atopic dermatitis in mice through improving intestinal microbiota. J Appl Microbiol. 2015;119(2):560-570.

58. Matsumoto M, Ebata T, Hirooka J, et al. Antipruritic effects of the probiotic strain LKM512 in adults with atopic dermatitis. Ann Allergy Asthma Immunol. 2014;113(2):209-216.

59. Chang YS, Trivedi MK, Jha A, Lin YF, Dimaano L, García-Romero MT. Synbiotics for prevention and treatment of atopic dermatitis: a metaanalysis of randomized clinical trials. JAMA Pediatr. 2016;170(3): 236-242.

60. Dotterud CK, Avershina E, Sekelja M, et al. Does maternal perinatal probiotic supplementation alter the intestinal microbiota of mother and child? J Pediatr Gastroenterol Nutr. 2015;61(2):200-207.

61. Louis P, Flint HJ, Michel C. How to manipulate the microbiota: prebiotics. Adv Exp Med Biol. 2016;902:119-142.

62. Moro G, Arslanoglu S, Stahl B, Jelinek J, Wahn U, Boehm G. A mixture of prebiotic oligosaccharides reduces the incidence of atopic dermatitis during the first six months of age. Arch Dis Child. 2006;91(10):814-819.

63. Boženský J, Hill M, Zelenka R, Skýba T. Prebiotics do not influence the severity of atopic dermatitis in infants: a randomised controlled trial. PLoS One. 2015;10(11):e0142897.

64. van der Aa LB, Heymans HS, van Aalderen WM, et al; Synbad Study Group. Effect of a new synbiotic mixture on atopic dermatitis in infants: a randomized-controlled trial. Clin Exp Allergy. 2010;40(5):795-804.

65. Grüber C, van Stuivenberg M, Mosca F, et al. Immunoactive prebiotics transiently prevent occurrence of early atopic dermatitis among low-atopy-risk infants. J Allergy Clin Immunol. 2015;136(6): 1696-1698.

66. Harima-Mizsawa N, Kamachi K, Kano M, et al. Beneficial effects of citrus juice fermented with Lactobacillus plantarum YIT 0132 on atopic dermatitis: results of daily intake by adult patients in two open trials. Biosci Microbiota Food Health. 2016;35(1):29-39.

67. van Nood E, Vrieze A, Nieuwdorp M, et al. Duodenal infusion of donor feces for recurrent Clostridium difficile. $N$ Engl J Med. 2013; $368(22): 407-415$
Clinical, Cosmetic and Investigational Dermatology

\section{Publish your work in this journal}

Clinical, Cosmetic and Investigational Dermatology is an international, peer-reviewed, open access, online journal that focuses on the latest clinical and experimental research in all aspects of skin disease and cosmetic interventions. This journal is included on PubMed. The manuscript management system is completely online

\section{Dovepress}

and includes a very quick and fair peer-review system, which is all easy to use. Visit http://www.dovepress.com/testimonials.php to read real quotes from published authors 